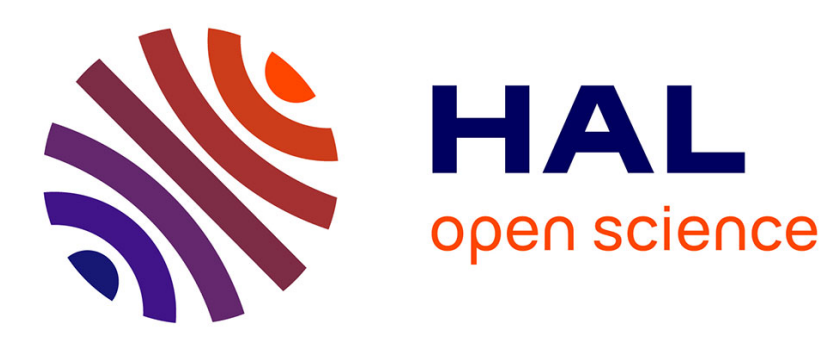

\title{
Organiser et conserver la mémoire de l'éphémère: les capsules de MemoRekall
}

Clarisse Bardiot

\section{To cite this version:}

Clarisse Bardiot. Organiser et conserver la mémoire de l'éphémère: les capsules de MemoRekall. Culture et Musées, 2017, 30, pp.159-174. hal-01843912

\section{HAL Id: hal-01843912 \\ https://hal.science/hal-01843912}

Submitted on 19 Jul 2018

HAL is a multi-disciplinary open access archive for the deposit and dissemination of scientific research documents, whether they are published or not. The documents may come from teaching and research institutions in France or abroad, or from public or private research centers.
L'archive ouverte pluridisciplinaire HAL, est destinée au dépôt et à la diffusion de documents scientifiques de niveau recherche, publiés ou non, émanant des établissements d'enseignement et de recherche français ou étrangers, des laboratoires publics ou privés. 


\title{
ORGANISER ET CONSERVER LA MÉMOIRE DE L'ÉPHÉMÈRE : LES CAPSULES DE MEMOREKALL
}

\author{
CLARISSE BARDIOT \\ UNIVERSITE DE VALENCIENNES
}

\begin{abstract}
Résumé
À la suite de l'initiative pionnière de la Tate Modern en 2004, plusieurs musées de renommée internationale se sont dotés d'un département intitulé time-based media art. Celui-ci regroupe des œuvres se caractérisant par leur durée. Parmi elles, des performances, dont la conservation repose sur la documentation. Parmi les documents, la captation vidéo occupe une place à part, par l'importance qui lui est accordée et par les nombreuses critiques qui lui sont faites. Les technologies numériques proposent deux types d'approches pour pallier aux biais de la captation : intra-documentaire (annoter la captation) et inter-documentaire (connecter la captation à un corpus documentaire plus vaste). Après avoir développé ces deux stratégies au travers d'exemples récents, nous présentons MemoRekall, une application web qui permet de les réunir au sein d'une même interface homme-machine. L'agencement des ressources documentaires et des annotations crée un nouveau document, une capsule. Celle-ci organise la documentation et propose une stratégie de conservation des œuvres. L'objectif est de déduire l'intention artistique de l'articulation des documents et d'aider ainsi à établir l'authenticité d'une ré-interprétation via la documentarisation.
\end{abstract}

\section{Mots-clés}

Documentation, conservation, arts de la scène, interface homme-machine, time based media art

En ce début de millénaire, la performance et les arts de la scène, et en particulier la danse, investissent ou plutôt réinvestissent les espaces d'exposition et les musées. C'est ainsi qu'en 2008, la Ferme du Buisson (Marne-la-Vallée) a présenté une manifestation au titre explicite : Une exposition chorégraphiée. Comme l'a montré Claire Bishop, le phénomène n'est pas nouveau : deux vagues similaires l'ont précédé, au tournant des années 1930-1940 puis 19601970 (Bishop, 2014 : 63). La scène se déplace vers le musée ou la galerie, de la «black box » au «white cube ». L'un des exemples les plus récents est Work/Travail/Arbeid (2015) d'Anne Teresa De Keersmaeker s'étirant pendant neuf semaines au Wiels (Bruxelles). Quatorze danseurs et six musiciens œuvrent pendant la totalité des heures d'ouverture du musée. Une version réduite, de neuf jours, a été présentée au Centre Pompidou (Paris) et à la Tate Modern (Londres) en 2016 avant de nouvelles dates prévues en 2017 au MoMA (New York).

Dans ce contexte, en 2004, la Tate Modern se dote d'un nouveau département intitulé timebased media art, qui regroupe des œuvres se caractérisant par leur durée. Entrent dans les collections des créations dont la dimension temporelle prévaut sur la dimension spatiale. Cette reconnaissance est un infléchissement du musée comme lieu de conservation exclusif des arts de l'espace. Dans ce glissement de l'espace au temps, de nouveaux objets intègrent les collections muséales, parmi eux les performances, aux côtés d'installations vidéo ou d'œuvres 
numériques. La complexité et la nouveauté des défis de conservation engendrés par cette nouvelle catégorie d'œuvres est à l'origine de la création de départements dédiés. Aujourd'hui, à la suite de la Tate Modern, une dizaine de musées se sont dotés d'un département timebased media art. L'institution britannique acquiert en 2004 sa première performance, Good Feelings in Good Times, de l'artiste Roman Ondák (2003). Après l'ouverture du Tanks en 2012, présenté comme le premier espace muséal permanent dédié aux arts vivants, la Tate inaugure en juin 2016 une nouvelle extension avec trois semaines de performances issues de sa collection, laquelle s'est étoffée en douze ans d'acquisitions. Pourtant, objet immatériel, éphémère et non reproductible, la performance est considérée comme impossible à collecter et collectionner. Seules restent les traces (photographies, captations vidéo, diagrammes, objets...), les reliques, acquises par les musées et parfois élevées au rang d'œuvres d'art. Soit la documentation mais non l'œuvre elle-même dont l'ontologie résiderait dans le hic et nunc de la représentation et la coprésence des acteurs et des spectateurs. Peggy Phelan, auteure d'un ouvrage majeur sur la performance, est sans appel : "Performance cannot be saved, recorded, documented, or otherwise participate in the circulation of representations of representations: once it does so it becomes something other than performance. » (Phelan, 1993 : 146.)

L'inscription de la performance et de la danse dans l'espace muséal pose de nouveaux défis quant à leur conservation : comment préserver une œuvre par nature éphémère, réputée attachée au corps de l'artiste, pour l'intégrer dans une collection permanente et être en mesure de la re-présenter au public ? Quels protocoles mettre en œuvre, quelles conditions réunir pour faire en sorte de répéter à l'infini «ce qui ne reste pas » (Van Imschoot, $2005: 108$ ) ? Comment organiser et conserver la mémoire de l'éphémère ? Ces questions sortent du cadre du musée, même si le contexte que nous venons de décrire leur confère davantage d'acuité dans ce contexte précis. Elles concernent l'ensemble des arts de la scène, tant la possibilité du répertoire, de la reprise ou de la reconstitution. Ces trois termes ne désignent pas exactement les mêmes pratiques, ni les mêmes temporalités. Le répertoire s'ancre dans le patrimoine, soit un ensemble d'œuvres interprétées au cours du temps - parfois plusieurs dizaines d'années par différentes équipes artistiques, comme pour les ballets de danse classique. La reprise a lieu au sein d'une même compagnie, à quelques mois ou quelques années de distance, afin par exemple de prolonger la durée d'exploitation d'une pièce, avec des adaptations de la version originale, adaptations dont la nature et l'importance sont variables : changement d'interprète («reprise de rôle»), modification du plan de feu ou de l'ordre des séquences, suppression d'une scène, transformation de la scénographie, etc. La reconstitution est un spectacle historique rejoué, réinterprété par une compagnie contemporaine, sur la base de traces documentaires.

\section{DOCUMENTER POUR PRÉSERVER}

À la suite des problématiques que nous venons d'évoquer, la Tate Modern a mis en place de 2012 à 2013 le programme de recherche "Collecting the Performative ; a Research Network Examining Emerging Practice for Collecting and Conserving Performance Based Art » sous la direction de Pip Laurenson, conservateur au musée, en collaboration avec la chercheure Vivian van Saaze. Dans la lignée de Claire Bishop et du concept de «performance déléguée $»^{1}$ (Bishop, 2012 : 219-240), Pip Laurenson et Vivian Van Saaze considèrent que les performances peuvent entrer dans les collections à partir du moment où elles sont

${ }^{1}$ Le chapitre sur la performance déléguée a été traduit en français in Bardiot, Clarisse, et Romaric Daurier, éd. 2013. Black / White / Live Box. Cabarets de curiosités 2. Valenciennes: Subjectile ; Le Phénix. 
désolidarisées de la présence de l'artiste. Il faut pouvoir détacher l'œuvre du corps de son auteur même :

«Phelan voices a view about performance art that is no longer true for all performance works. [...] The types of performance works which enter collections as live works can exist, at least theoretically, independent of the artist and can be repeated or re-activated in the future. In this sense they are durable and portable. Within current practice, artists are themselves finding formats that allow works which are not straightforward objects to be bought and sold, for example, by the use of scripts or instructions which enable others to perform and reperform the work. Unlike many performances from the 1960s and 1970s, recent performance artworks often no longer privilege the live moment or the artist's own body. » (Laurenson \& Van Saaze, 2014 : 32-33.)

Dans ce contexte, la documentation joue un rôle crucial. Sans elle, impossible de re-présenter une œuvre, de la reprendre ou de la reconstituer. Pip Laurenson et Vivian van Saaze mentionnent ci-dessus l'exemple des scripts et des instructions, faisant office de partitions. Mais il ne s'agit pas de la seule démarche possible. Anne Bénichou, professeure à l'UQÀM (Montréal) réunit dans un ouvrage collectif les différentes options mises en œuvre actuellement et résume ainsi la situation :

« Deux opérations sont privilégiées, la documentation et la reprise, non pour les envisager comme des étapes ou des modalités différentes ou consécutives de la transmission (l'archive d'un côté et le live de l'autre), mais pour penser leurs liens étroits, leur enchevêtrement dans l'entreprise de transmission. Plusieurs théoriciens des performances studies et de la danse contemporaine ont récemment insisté sur l'importance de réarticuler l'archive et le vivant, la documentation et la reprise, le script et la recréation. [..] Les notations, les partitions et les scripts d'œuvres performatives et chorégraphiques suscitent un intérêt accru [...]. Quant aux documents et aux archives, ils sont pensés non plus seulement en tant que traces, mais aussi en tant que scripts permettant de réactualiser les œuvres dont ils sont issus, ce glissement suscitant de nouvelles herméneutiques de la documentation. » (Bénichou, 2015 : 10-11.)

Dans ces nouvelles interprétations sémiotiques, la captation vidéo demeure une trace majeure pour la documentation et la préservation des œuvres, même si les travaux précédemment cités ne lui accordent pas de traitement spécifique. La captation vidéo a été, avant la capture de mouvement, la réponse à l'archivage de ce document qu'est le corps de l'interprète. Provoquant maints débats depuis les années 60, souvent considérée comme une «trahison » de l'œuvre originale (Melzer, 1995a ; 1995b), elle s'est aujourd'hui banalisée. La captation vidéo est devenue un document parmi d'autres, aux côtés des partitions, des carnets de notes, des photographies, des croquis de scénographies, des documents de production, etc. Ce nouveau statut est également à mettre sur le compte de la banalisation de la pratique : aujourd'hui tout est filmé, notamment depuis que les téléphones portables sont équipés de caméras. Le rôle des technologies, et en particulier les possibilités offertes par les smartphones, dans la manière de documenter ainsi que dans les documents produits, ne doit pas être sous-estimé.

La captation vidéo est un document à la fois essentiel et parcellaire : essentielle, elle enregistre les corps en mouvement et par ce biais transforme les spectacles en document; parcellaire, elle ne donne qu'un seul point de vue, ne présente qu'une partie de ce qui a lieu sur scène, engendre des collusions temporelles par le biais du montage, adapte l'éclairage aux conditions de luminosité des caméras. Enfin, comme le souligne Anne Bénichou (cf. supra), elle n'est pas seulement une trace : elle participe aussi de l'œuvre, intervenant dans son processus de création (répétitions filmées), servant d'appui aux reprises ou à sa promotion.

Pour pallier les insuffisances ou les biais de la captation vidéo, deux types de stratégies sont développées: inter-documentaire (connecter la captation à un corpus documentaire plus 
vaste) et intra-documentaire (annoter la captation). Les technologies numériques permettent des approches novatrices de ces deux stratégies et nous en présenterons des exemples dans les lignes qui suivent. Enfin, nous proposons de conjuguer ces deux approches, interdocumentaire et intra-documentaire, dans un seul outil: MemoRekall, une application numérique dédiée à la documentation des arts de la scène en vue de leur conservation. En comparaison du traitement traditionnel effectué par les institutions qui en ont la charge, les différentes démarches évoquées ici proposent une organisation et une mise à disposition originales des archives.

\section{L'APPROCHE INTRA-DOCUMENTAIRE : \\ ANNOTER LA CAPTATION VIDÉO}

La captation vidéo est rarement autosuffisante et appelle le commentaire, l'analyse et le décryptage. Ce constat recoupe notre expérience d'enseignante et de chercheure : il est quasiment impossible de retransmettre à un public d'étudiants la nature d'une performance par le seul document vidéo. Systématiquement, nous sommes amenée à faire des pauses dans l'enregistrement lors d'une seconde lecture afin d'expliciter, préciser, compléter. De même, travaillant à l'analyse de processus de création d'artistes contemporains, il est frappant de constater leur besoin d'intervenir auprès du chercheur lors de sa consultation des archives vidéo. Ces interventions sont autant d'annotations orales de l'image en mouvement, que l'on retrouve aujourd'hui, grâce aux outils numériques, sous forme d'annotations textuelles, sonores ou graphiques ${ }^{2}$.

Comme le développe le récent numéro spécial de Performance Research intitulé On An/Notations (2015), le phénomène est loin d'être marginal et concerne non seulement la pratique des chercheurs mais aussi celle des éditeurs, des artistes et du public. Pour les chercheurs en danse Scott Delahunta, Kim Vincs et Sarah Whatley, qui ont coordonné ce numéro, "there are no strict distinctions between annotation and notation as markmarking when marking is an extension of thinking, not merely a recording » (Delahunta, Vincs \& Whatley, 2015 : 2). Un exemple récent de cette approche, où notation et annotation sont étroitement intriquées, est la publication numérique A Steady Pulse, à l'occasion de la reprise des «danses en silence» de Lucinda Childs. Un split-screen (écran fractionné) permet de visualiser simultanément la captation vidéo de Melody Excerpt (1977) dans sa réinterprétation de 2013 et la partition graphique de l'œuvre animée (Bissel, 2015 : s.p.). L'animation est à la fois une notation et une annotation de la captation.

L'annotation, pour reprendre la définition de Manuel Zacklad, désigne «toute forme d'ajout visant à enrichir une inscription ou un enregistrement pour attirer l'attention du récepteur sur un passage ou pour compléter le contenu sémiotique par la mise en relation avec d'autres contenus sémiotiques préexistants ou par une contribution originale » (Zacklad, 2007 : 34). Elle est intra-documentaire au sens où, en marge du document - ici la captation vidéo -, elle en est solidaire tout en proposant un enrichissement, un développement, un complément sur un point ou une séquence précise. L'annotation relève de la lecture de près (close reading) avec la possibilité d'un grand degré de précision.

Plusieurs initiatives pour créer des applications dédiées aux besoins spécifiques des arts de la scène se sont développées récemment (Bardiot, 2015a ; 2015b). Elles sont à la fois le fait d'institutions conservant des fonds dédiés au spectacle vivant, de recherches universitaires

\footnotetext{
${ }^{2}$ Preuve de l'importance de l'annotation dans un contexte numérique, le W3C a établi en 2014 un groupe de travail officiel afin de définir un standard pour les annotations sur le web. https://www.w3.org/annotation/
} 
consacrées à la documentation des arts de la scène ou encore d'équipes artistiques. Souvent, ces trois acteurs sont amenés à collaborer au sein de programmes multidisciplinaires.

L'un des axes explorés est la création de logiciels d'annotation vidéo pour le spectacle vivant. Dans le cadre des projets de documentation numérique mis en œuvre par William Forsythe (Bardiot, 2015 c), l'un des danseurs de sa compagnie, David Kern, est à l'origine de Piecemaker, initialement dédié aux danseurs pendant le temps des répétitions. Piecemaker évolue et devient PM2GO à l'occasion du projet Motion Bank, en 2014. Cette application hors ligne permet d'annoter textuellement les captations de répétitions ou de spectacles, en différé ou en direct. L'interface est divisée en trois parties: la vidéo, les «Cues» (marqueurs d'entrée et de sortie d'une annotation) et les annotations elles-mêmes. Celles-ci peuvent être regroupées avec des codes couleur. Plusieurs personnes peuvent annoter une même captation.

L'annotation de captations vidéo regroupe de nombreuses approches, témoignant d'un rapport à l'archive et au document loin d'être univoque : transcription et chapitrage des répétitions ou de l'œuvre; réalisation des annotations par un chercheur (En scènes de l'INA), le chorégraphe, un danseur ou un membre de l'équipe artistique (Piecemaker), ou encore le public (MyStoryPlayer, NYPL Digital Collections, Siobhan Davies Replay) ou une équipe multidisciplinaire rassemblant chercheurs et artistes (Synchronous objects de William Forsythe, Maria Palazzi et Norah Zuniga Shaw); utilisation d'annotations textuelles (En scènes, Spectacles en ligne de l'IRI - Institut de recherche et d'innovation du Centre Pompidou), graphiques (RotoSkecht de Zachary Lieberman, Scott deLahunta et Susan Rethorst), graphiques et sonores (Improvisation Technologies de William Forsythe) ou les trois modes réunis (Creation-Tool du projet TKB mené par Carla Fernandes, Synchronous objects) ; publication des annotations sur internet de manière publique (Motion Bank) ou dans un espace privé (Siobhan Davies Replay), via une publication type DVD (Capturing intention, sur l'œuvre d'Emio Greco) ou sur son disque dur (PM2GO); utilisation d'outils disponibles dans d'autres contextes (Advene, AmiGram, Anvil, ELAN, On the mark, SSI, Vcode/VData...) ou bien développés spécifiquement; annotations à destination publique ou personnelle; annotations en vue d'une explicitation, d'une contextualisation ou bien pour modifier la création initiale voire susciter une nouvelle œuvre, etc.

\section{L'APPROCHE INTER-DOCUMENTAIRE :}

\section{CONNECTER LA CAPTATION VIDEO}

L'annotation vidéo constitue une démarche intra-documentaire. La captation suscite également une autre approche, qualifiée d'inter-documentaire, selon la définition de Suzanne Briet: "La documentation, lorsqu'elle est intimement liée à la vie d'une équipe de travailleurs, ou scientifiques, ou érudits, - ou lorsqu'elle participe à une activité industrielle, commerciale, administrative, enseignante, etc ..., peut dans certains cas aboutir à une création véritable, par juxtaposition, sélection et comparaison de documents et production de document auxiliaires. Le contenu de la documentation est alors interdocumentaire. ${ }^{3}$ (Briet, 1951 : 13.) Dans le cas de la captation, l'approche inter-documentaire consiste à connecter le document vidéo à un ensemble d'autres documents (textes, images, sons, en ligne ou hors ligne). Ceux-ci permettent de combler les lacunes de la captation, d'élargir le cadre de l'image au contexte, au processus de création, à la parole de toute l'équipe artistique, à la réception du public, de préciser des aspects qui ne sont qu'évoqués. La captation ne devient pleinement intelligible qu'au sein d'un écosystème documentaire qui permet d'établir des correspondances, de corréler les informations, de mailler les traces, ou d'en révéler le contenu sémiotique. L'approche inter-documentaire souligne l'interdépendance entre les documents

\footnotetext{
3 Anne Bénichou fait également référence à la documentation interdocumentaire dans sous ouvrage (Bénichou, 2015 : 129).
} 
d'un même corpus ou dossier documentaire. Ces derniers ne peuvent uniquement se composer d'un ensemble de pièces cohérentes: il faut également établir des relations entre les documents. La captation vidéo ne saurait être la source unique de la documentation des arts de la scène, la trace suprême de ce qui a eu lieu.

Les Dance Capsules des spectacles de Merce Cunningham et les Choreographer's Score d'Anne Teresa De Keersmaeker sont deux exemples récents relevant d'une approche interdocumentaire telle que nous venons de la définir, avec des objectifs différents : transmettre les œuvres (M. Cunningham) et analyser une démarche artistique (A. T. De Keersmaeker).

Les Dance Capsules, réalisées par le Merce Cunningham Trust, concernent quatre-vingt-six chorégraphies des années 1940 à la fin de la vie du chorégraphe. Ce travail a été conduit dans un souci revendiqué de transmission des œuvres, alors que $\mathrm{M}$. Cunningham décide que sa compagnie sera dissoute deux ans après sa mort. La page d'accueil du site internet sur lequel les capsules sont accessibles (en partie librement, en partie avec une demande d'autorisation) précise leur objectif : faire en sorte que «les générations futures puissent étudier et jouer ces œuvres en sachant comment elles ont été initialement créées ${ }^{4}$ (Merce Cunningham Trust, 2016 : s.p.). Chaque capsule contient de vingt à quatre-vingts documents et plus : captations vidéo des représentations et des répétitions, plans de feux, dessins des costumes, interviews avec des danseurs et des membres de l'équipe artistique, photographies de la scénographie, extraits des carnets de notes de M. Cunningham, notes de production, musique originale, programmes, revue de presse, crédits, distribution, etc. Aucun lien ne semble établi a priori entre les documents : il s'agirait pour chaque spectacle d'une collection dont les éléments sont regroupés par catégories, avec une mise en exergue des documents vidéo, souvent accessibles sans autorisation et apparaissant en tête dans la liste des documents disponibles. D'une part, le terme de capsule lui-même suggère une cohérence : chaque ensemble renferme, contient des documents qui se répondent les uns aux autres et oriente la lecture de l'œuvre. En effet, chaque capsule ne comprend pas l'ensemble des archives, mais une sélection de documents, effectuée en vue de la transmission des œuvres vers d'autres compagnies. D'autre part, le Merce Cunningham Trust, éditeur des capsules, gère également les licences pour s'assurer que toute reprise s'effectue dans le respect de la création originale : les anciens danseurs de la compagnie sont chargés d'accompagner la dissémination des archives auprès d'autres danseurs et leur mémoire corporelle, l'archive vivante qu'ils sont devenus, effectue le lien entre les documents.

La publication d'une série de trois ouvrages récents d'Anne Teresa de Keersmaeker (DE Keersmaeker \& Cvejić, 2012 ; De Keersmaeker \& Cvejić, 2013 ; De Keersmaeker \& Cvejić, 2014) intitulée $A$ Choreographer's Score repose sur la complémentarité entre les supports. Le premier concerne les œuvres de jeunesse (Fase, Rosas danst Rosas, Elen's Aria et Bartók), le deuxième un diptyque (En Atendant et Cesena) créé au festival d'Avignon en 2010 et 2011, le troisième deux chorégraphies sur une musique de Steve Reich, Drumming (1998) et Rain (2001). Si leur facture est légèrement différente, les trois ouvrages s'articulent autour de DVD (des extraits des captations vidéo des œuvres étudiées ainsi que les entretiens filmés) et d'un livre, le tout réuni dans un coffret. Chacun des livres repose sur la transcription annotée et enrichie des entretiens présentés dans les DVD. Ils sont complétés par d'autres documents : croquis, photographies, articles de presse, notes de répétitions, etc. Au lecteur de choisir sa porte d'entrée puis de tisser les liens, de commencer par la vidéo et de poursuivre par la lecture des textes et des documents, ou vice versa, et appréhender ainsi la mise en partition des spectacles au travers d'un corpus documentaire multimodal. Dans ce projet éditorial, la transmission des œuvres vers d'autres danseurs n'est pas l'objectif premier. L'ensemble

\footnotetext{
${ }^{4}$ Notre traduction.
} 
démontre la complémentarité des supports et des mémoires (corporelle, visuelle, textuelle) pour saisir la démarche d'Anne Teresa de Keersmaeker et mener une analyse approfondie des chorégraphies.

Ces deux exemples reposent sur des captations vidéo de grande qualité et concernent des chorégraphes de premier plan très soucieux de la documentation de leur œuvre. Ils démontrent à quel point la captation est loin d'être une trace autosuffisante. Elle doit s'articuler avec une approche inter-documentaire. Au lecteur de réagencer les documents, de les articuler les uns par rapport aux autres, de trouver son propre chemin au travers d'un corpus hétéroclite et interdépendant, au travers d'un «dossier» dont la captation peut éventuellement être un élément saillant, mais jamais autonome.

\section{LES CAPSULES DE MEMOREKALL}

Approches inter-documentaire et intra-documentaire ne sont pas exclusives l'une de l'autre et la frontière est parfois poreuse. Complémentaires, ces deux approches peuvent être utilement combinées et jouer simultanément de différents rapports au document, entre approfondissement et connexion. Elles offrent différentes strates de navigation à l'intérieur et à l'extérieur de la captation vidéo. Mettre sur un même plan des documents numérisés et nativement numériques au sein d'une même interface homme-machine est propice à la réappropriation des corpus documentaires non seulement par les artistes et leurs équipes, par les conservateurs, mais aussi par le public.

Dans le contexte d'un projet plus vaste (Rekall), et avec le souhait de prolonger mes réflexions sur l'ingénierie documentaire liée à la conservation des arts de la scène via l'instrumentation informatique et le développement d'interfaces homme-machine, nous avons conçu en collaboration avec Guillaume Marais, Guillaume Jacquemin et Thierry Coduys une application en ligne, MemoRekall ${ }^{5}$, mise en ligne en septembre 2015. Elle est à la fois un espace d'écriture, en mode édition, et un espace de lecture, en mode consultation. Open source et gratuite, MemoRekall permet d'enrichir les captations vidéo en conjuguant les démarches inter et intra-documentaire : elle conjugue annotation et connexion à d'autres ressources documentaires. Le résultat est une «capsule» (nous empruntons le terme à $\mathrm{M}$. Cunningham) qu'il est possible d'intégrer à n'importe quelle page web. La capsule comprend la captation ainsi que l'ensemble des annotations et des documents liés (ou des liens lorsqu'il s'agit de ressources en ligne). Elle offre une lecture augmentée de la captation au sein d'un environnement multimodal et peut être actualisée à volonté. MemoRekall se situe au carrefour de la valorisation de documents culturels numériques existants, de la création de contenus culturels enrichis et de la mise en place d'espaces critiques et collaboratifs.

MemoRekall propose une lecture multi-documents, temporelle et spatiale, d'une œuvre, à partir de son enregistrement vidéo. Si cette application peut être utilisée dans de nombreux contextes, elle a avant tout été créée pour les besoins spécifiques du time-based media art et tout particulièrement des arts de la scène. La conception d'interfaces numériques ad hoc permet d'étendre la connaissance et la compréhension du spectacle vivant. L'agencement des ressources documentaires et des annotations crée un nouveau document, une capsule. Celle-ci organise la documentation et propose une stratégie de conservation des œuvres.

Partant du principe que la captation était l'une des traces majeures, et quasiment systématique dans le processus de documentarisation des arts de la scène, mais que cette trace appelait à la fois le commentaire et le complément d'information via des documents annexes, nous avons choisi d'en faire l'épine dorsale de MemoRekall. L'organisation des annotations et des

\footnotetext{
${ }^{5}$ MemoRekall a reçu le soutien du Ministère de la Culture et de la Communication (appel à projet services culturels innovants) et de nombreuses institutions culturelles.Publication en ligne : < www.memorekall.fr〉. Consulté le 11 novembre 2017.
} 
documents s'élabore à partir et autour de la captation vidéo dans une logique hypertextuelle. Alors que, dans les exemples de M. Cunningham ou de A. T. De Keersmaeker, le lecteur pouvait choisir son cheminement dans n'importe quel ordre documentaire, ici, il doit commencer par l'enregistrement filmique de la représentation. Au fur et à mesure de la consultation, le lecteur découvre annotations et autres documents, qu'il peut ouvrir et consulter à sa guise, pour revenir ensuite au film. De même, l'auteur de la capsule doit commencer en premier lieu par choisir un document vidéo et l'importer dans l'application. Le temps structure l'ordre d'apparition documentaire, même si, comme nous allons le voir dans la description de l'interface, il est également possible d'accéder aux différents contenus par leur organisation spatiale dans l'écran et de s'affranchir ainsi de toute chronologie imposée par l'auteur de la capsule.

L'interface comprend quatre espaces principaux (figure 1): à gauche, une barre de menu, avec des boutons pour ajouter des notes, des documents ou des liens vers des ressources en ligne, ainsi que quelques fonctions de paramétrages (choix d'un auteur et d'un titre, copyright, export en xml, code pour l'intégration dans une page web); au centre, dans l'espace supérieur, une place importante est réservée à la captation vidéo ; dans la partie inférieure, une timeline (plan de montage chronologique) présente les points d'entrée des annotations et des documents connectés ; à droite, une colonne rassemble l'ensemble des documents liés par ordre chronologique. À la lecture de la vidéo, les annotations apparaissent en surtitrage, et les documents liés s'éclairent. Le créateur de la capsule leur affecte un timecode (code horaire) et une durée. Celle-ci est visible grâce à une barre de temps qui s'affiche sous le document. Si pour le moment les annotations sont uniquement textuelles, les documents peuvent être de n’importe quelle nature : textes, images, sons, vidéos. Ils sont téléchargés par le créateur de la capsule sur le serveur de MemoRekall. Les ressources en ligne sont disponibles sous forme de liens hypertextes et ne sont pas copiées sur le serveur de MemoRekall, dans le respect des droits d'auteur. Documents téléchargés et liens internet peuvent être annotés dans une fenêtre en рор ир (fenêtre contextuelle). Les manipulations s'opèrent directement dans le navigateur internet et sont sauvegardées. Il est possible de contribuer, seul ou à plusieurs, à la création d'une captation enrichie.

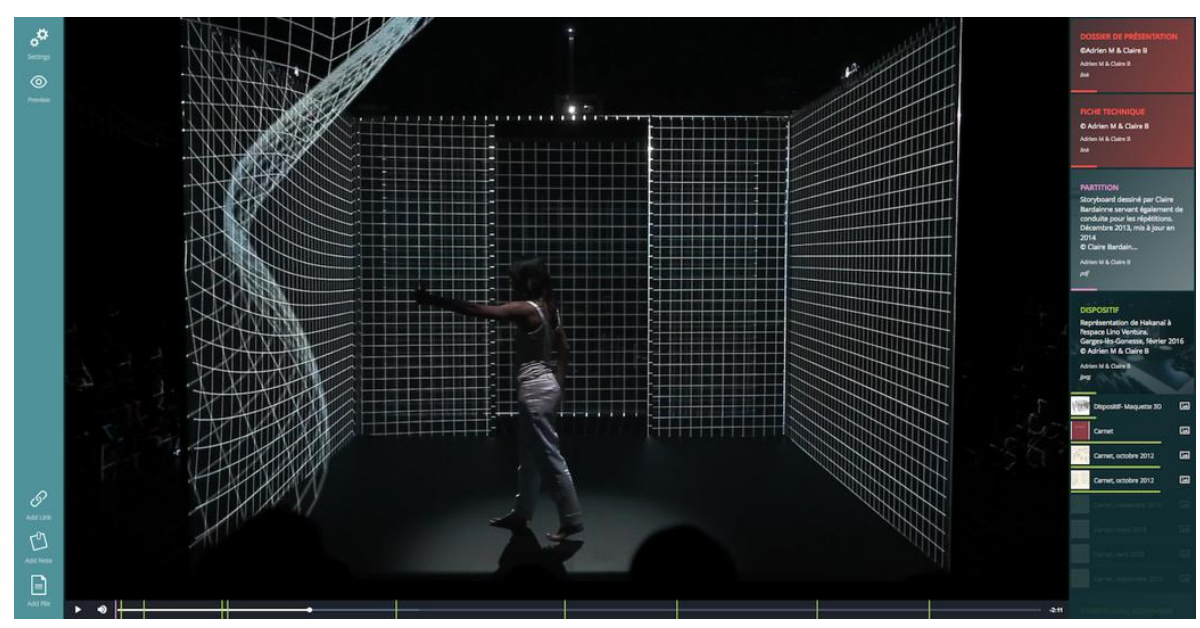

Figure 1 : capture d'écran de l'interface de MemoRekall en mode édition.

L'œuvre analysée est Hakanaï d'Adrien M \& Claire B. Capsule réalisée par Clarisse Bardiot pour l'édition numérique de l'ouvrage La neige n'a pas de sens. Adrien $M \&$ Claire B, Valenciennes, (C) Subjectile, 2016. 
À partir d'une même vidéo, différentes stratégies documentaires peuvent être développées, en fonction des enjeux propres à chaque auteur de capsule. Nous avons utilisé MemoRekall dans des classes pour familiariser des élèves (collège, lycée et université) avec l'analyse de spectacle, dans des théâtres pour la médiation numérique des œuvres auprès de différents publics, auprès de chorégraphes et de metteurs en scène pour documenter leur processus de création ou un spectacle achevé. MemoRekall offre de nombreuses possibilités de scénarios d'usages : documenter pour la reprise, la transmission d'un répertoire ou la reconstitution ; pour une exposition/publication en ligne ; pour accompagner la diffusion de l'œuvre ; pour différents bénéficiaires, privés, collectifs ou publics. En fonction de chaque scénario, le statut et la nature des annotations comme des documents liés sont différents. MemoRekall est à la fois un outil de création, de conservation, de critique d'art et de publication.

\section{CONCLUSION}

La documentation joue un rôle crucial dans la conservation des performances et des arts de la scène. Les technologies numériques permettent une réappropriation des contenus des documents, et en particulier de la captation vidéo qui jouit d'un statut à la fois privilégié et controversé. La conjugaison de deux approches, intra et inter-documentaire, permet de développer l'interprétation de la documentation en fonction des usages : conserver, diffuser, analyser, créer une œuvre. Dans le cas de la préservation des œuvres, le document retrouve ses « deux sens complémentaires : transmettre et prouver» (Salaün, 2012 : 36). Le document transmet des informations, des connaissances, afin que l'œuvre puisse être re-présentée ; il peut également devenir une preuve lorsque l'on cherche à établir si une réinterprétation est authentique ou non. Or l'authenticité d'une œuvre immatérielle est difficile à saisir en l'absence de la conservation d'un objet matériel. La lecture de la documentation, le fait que l'on puisse se référer non seulement aux traces mais aux connexions entre les traces, et à la sédimentation des annotations laissées par différents interprètes (les conservateurs, les commissaires d'exposition, les compagnies à qui la réinterprétation des œuvres est confiée) permettent d'établir l'authenticité d'une œuvre réinterprétée. L'intention artistique est plus difficile à saisir. La tentation est grande de considérer qu'il suffit de la lire dans les déclarations de l'artiste, dans les « notes d'intention » conservées dans le corpus, voire dans la lecture de l'œuvre elle-même. Il nous semble au contraire, et nous partageons cette analyse avec Vivian Van Saaze (Saaze, 2013), que l'intention artistique apparaît en filigrane dans l'analyse inter et intra-documentaire. Il ne s'agit pas d'un « message » délivré « en clair » par l'artiste, mais bien d'une enquête, d'une analyse et au final d'une interprétation qui doit être menée par la personne responsable de l'interprétation de l'œuvre. L'élaboration d'un outil numérique tel que MemoRekall, et la réalisation de capsules à des fins de conservation, ont pour ambition de déduire l'intention artistique de l'articulation des documents et d'aider ainsi à établir l'authenticité d'une réinterprétation via la documentarisation. 


\section{Bibliographie}

Bardiot (Clarisse). 2015 a. «Rekall : an environment for notation / annotation / denotation ». Performance Research 20 (6), p. 82-86.

Bardiot (Clarisse). 2015 b. «Video recording and documentation of scenic arts : from the annotation to the visualization of metadata, the example of the Rekall software », p. 159-68 in Acoustic Space. Data Drift. Archiving Media and Data Art in the 21st Century / sous la direction de Rasa Smite, Raitis Smits, et Lev Manovich, 14.

Bardiot (Clarisse). 2015 c. «Une autre mémoire : la chorégraphie des données. À propos des objets numériques développés par William Forsythe (Improvisation Technologies, Synchronous objects et Motion Bank)», p. 235-52, in Recréer / scripter - Mémoires et transmissions des cuvres performatives et chorégraphiques contemporaines / sous la direction d'Anne Bénichou. Nouvelles scènes. Dijon : Les presses du réel.

Bénichou (Anne) (sous la dir. de). 2015. Recréer / scripter - Mémoires et transmissions des œuvres performatives et chorégraphiques contemporaines. Les Presses du réel. Dijon.

Bishop (Claire). 2012. Artificial Hells: Participatory Art and the Politics of Spectatorship. London ; New York : Verso Books.

Bishop (Claire). 2014. « The Perils and Possibilities of Dance in the Museum: Tate, MoMA, and Whitney ». Dance Research Journal, 46 (3), p. 63-76.

Bissel (Bill) (sous la dir. de). 2015. A Steady Pulse : Restaging Lucinda Childs, 1963-78. Philadlphia: The Pew Center for Arts \& Heritage. Publication en ligne : http://danceworkbook.pcah.us/asteadypulse/. Consulté le 5 septembre 2017.

Briet (Suzanne). 1951. Qu'est-ce que la documentation ? Paris : Édit.

Copeland (Mathieu) (sous la dir. de). 2013. Chorégraphier l'exposition Choreographing exhibitions. Noisiel ; St. Gall ; Dijon : La Ferme du Buisson ; Kunst Halle Sankt Gallen ; les Presses du Réel.

De Keersmaeker (Anne Teresa) et Cvejić (Bojana). 2013. En atendant \& Cesena: $a$ choreographer's score. Bruxelles : Mercatorfonds, Rosas.

De Keersmaeker (Anne Teresa) et Cvejić (Bojana). 2014. Drumming \& Rain: $a$ choreographer's score. Bruxelles : Fonds Mercator : Rosas.

deLahunta (Scott), Kim (Vincs) et Sarah Whatley. 2015. «On An/Notations ». Performance Research 20 (6), p. 1-2.

Laurenson (Pip) et van Saaze (Vivian). 2014. "Collecting performance-based art: new challenges and shifting perspectives », p. 27-41, in Performativity in the Gallery. Staging Interactive Encounters / sous la direction de Outi Remes, Laura MacCulloch et Marika Leino. Bern : Peter Lang Verlag.

Melzer (Annabelle). 1995. "'Best Betrayal': the Documentation of Performance on Video and Film, Part 1 ». New Theatre Quarterly 11 (42), p. 147-57.

Melzer (Annabelle). 1995. «'Best Betrayal': the Documentation of Performance on Video and Film, Part 2 ». New Theatre Quarterly 11 (43), p. 259-76.

Merce Cunningham Trust. 2016. «Dance Capsules». Publication en ligne : http://www.mercecunningham.org/film-media/dance-capsules/. Consulté le 5 septembre 2017.

Phelan (Peggy). 1993. Unmarked: the Politics of Performance. London ; New York: Routledge. 
Saaze (Vivian Van). 2013. Installation Art and the Museum: Presentation and Conservation of Changing Artworks. Amsterdam University Press.

Salaün (Jean-Michel). 2012. Vu, lu, su : les architectes de l'information face à l'oligopole du Web. Cahiers libres. Paris : La Découverte.

Van Imschoot (Myriam). 2005. « Rests in pieces : partitions, notation et trace dans la danse ». Multitudes 21 (2), p. 107-16.

Zacklad (Manuel). 2007. «Annotation : attention, association, contribution », p. 29-46 in Annotations dans les Documents pour l'Action / sous la direction de Manuel Zacklad et Pascal Salembier. Paris : Lavoisier. 